\title{
A prospective randomized trial comparing homologous and autologous fibrin sealants for the control of alveolar air leak
}

\author{
${\text { Burcu Kilıç }{ }^{1} \text {, Ezel Erşen }}^{1}$, Ahmet Demirkaya ${ }^{2}$, H. Volkan Kara ${ }^{1}$, Nurlan Alizade ${ }^{1}$, Mehlika Isşcan $^{1}$, Kamil Kaynak $^{1}$, \\ Akif Turna ${ }^{1}$ \\ ${ }^{1}$ Department of Thoracic Surgery, Cerrahpasa School of Medicine, Istanbul University, Fatih, Istanbul, Turkey; ${ }^{2}$ School of Medicine, Istanbul \\ Acıbadem University, Istanbul, Turkey \\ Contributions: (I) Conception and design: B Kılıç, A Turna; (II) Administrative support: K Kaynak, A Turna; (III) Provision of study materials \\ or patients: B Kılıç; (IV) Collection and assembly of data: B Kılıç, HV Kara, N Alizade, E Erşen, A Demirkaya, M İşcan; (V) Data analysis and \\ interpretation: B Kılıç, A Turna; (VI) Manuscript writing: All authors; (VII) Final approval of manuscript: All authors. \\ Correspondence to: Burcu Kılıç, MD. Cerrahpaşa Tıp Fakultesi, Cerrahi Bilimler Binası, 2 Kat, Fatih, Istanbul, Turkey. Email: burcu.aksoy@gmail.com.
}

Background: Postoperative air leak is a common complication seen after pulmonary resection. It is a significant reason of morbidity and also leads to greater hospital cost owing to prolonged length of stay. The purpose of this study is to compare homologous sealant with autologous one to prevent air leak following pulmonary resection.

Methods: A total of 57 patients aged between 20 and 79 (mean age: 54.36) who underwent pulmonary resection other than pneumonectomy (lobar or sublobar resections) were analyzed. There were 47 males (83\%) and 10 females (17\%). Patients who intraoperatively had air leaks were randomized to receive homologous (Tisseel; n=28) or autologous (Vivostat; $n=29$ ) fibrin sealant. Differences among groups in terms of air leak, prolonged air leak, hospital stay, amount of air leak were analyzed.

Results: Indications for surgery were primary lung cancer in 42 patients (71.9\%), secondary malignancy in 5 patients $(8.8 \%)$, and benign disease in 10 patients $(17.5 \%)$. Lobectomy was performed in 40 patients (70.2\%), whereas 17 patients $(29.8 \%)$ had wedge resection. Thirteen $(46.4 \%)$ patients developed complications in patients receiving homologous sealant while 11 (38.0\%) patients had complication in autologous sealant group $(\mathrm{P}=0.711)$. Median duration of air leak was 3 days in two groups. Time to intercostal drain removal was 3.39 and 3.38 days in homologous and autologous sealant group respectively $(\mathrm{P}=0.978)$. Mean hospital stay was 5.5 days in patients receiving homologous sealant whereas it was 5.0 days in patients who had autologous agent $(\mathrm{P}=0.140)$. There were no significant differences between groups in terms of measured maximum air leak $(\mathrm{P}=0.823)$ and mean air leak $(\mathrm{P}=0.186)$. There was no significant difference in the incidence of complications between two groups $(\mathrm{P}=0.711)$.

Conclusions: Autologous and heterologous fibrin sealants are safe and acts similarly in terms of air leak and hospital stay in patients who had resectional surgery.

Keywords: Air leak; fibrin sealant; thoracic surgery; Tisseel; Vivostat

Submitted Nov 21, 2016. Accepted for publication Jul 17, 2017.

doi: $10.21037 /$ jtd.2017.08.02

View this article at: http://dx.doi.org/10.21037/jtd.2017.08.02

\section{Introduction}

Postoperative air leak is a frequent complication after pulmonary resections. It is an important morbidity and it results in prolonged hospital stay and greater hospital cost (1).
Development of modern surgical methods requires the effective control of air leak following pulmonary resection. The preferred method to reduce air leaks is to prevent them from occurring; therefore, every measures should be taken to perform an appropriate dissection of the structures 
along correctly-defined anatomical planes between lobes and segments. In addition to suture or stapler applications, a number of various types of sealant have been developed to reduce or stop postoperative air leak, including collagen fleeces, fibrin sealants (2). Homologous fibrin glue received approval from Food and Drug Administration (FDA) for bleeding control, creating barrier and tissue adhesion and it is the only material used in the United States. It is a two component material that involves fibrin glue, concentrated fibrinogen and thrombin. This material is clinically useful for all fields of surgery departments and its efficacy and unwanted side effects has been researched in many studies (3).

It was reported that application of a new protocol about the chest tube removal by using digital air leak monitorization enabled receiving objective, reliable and appropriate information about air leak, and reduced the length and cost of hospital stay comparing to conventional chest tube removal method depending on instant subjective evaluation of air leaks $(4,5)$.

Our aim was to perform a randomized and prospective study which compares the effectiveness of autologous sealant with FDA-approved homologous fibrin tissue adhesives applied following pulmonary resection in terms of tube removal times (TRT), air leak, hospital stay.

\section{Methods}

This study was done between August 2010 and November 2015. A total of 368 consecutive patients who underwent lung resections were included in the study. Inclusion criteria included; being older than 17 years of age, undergoing lung resection (segmentectomy, wedge resection, lobectomy or bilobectomy), air leak observed following resection. Exclusion criteria were: prior thoracic surgery, prior pleurodesis. Air leakage was observed following resection in 80 of $360(22.2 \%)$ patients. Type-I $(\alpha)$ error and typeII ( $\beta$ ) error were set to $10 \%$. The study has been designed as a non-inferiority study to compare the effectiveness of autologous sealant with FDA-approved homologous sealant. Our non-inferiority margin was $40 \%$ difference of rate of prolonged air leak as a primary outcome with a $\delta$ value of $10 \%$. With a one-sided alternative, we needed to include 40 patients in each arm. Therefore, 80 patients were included in the study. In patients who underwent autologous fibrin sealant, eleven patients were excluded from the study because of postoperative pleurodesis implementation $(n=7)$ and technical failure of digital drainage system $(n=4)$. In autologous sealant group, 8 and 4 patients were excluded from the study because of the technical problems and pleurodesis respectively (CONSORT diagram) (Figure 1). A total of 57 patients were left randomized whom autologous $(\mathrm{n}=29)$ and homologous $(\mathrm{n}=28)$ fibrin glues were used following pulmonary resection (Figure 2). Randomization was performed according to an internet based-random number generator. Forty-seven patients $(82.5 \%)$ were male and ten patients $(17.5 \%)$ were female. Air leak that continued at least on the postoperative 7th day have been described as 'prolonged air leak'. TRT was determined as the last day when tube or tubes were removed postoperatively. The treatment groups were compared in terms of PAL (primary outcome) and TRT (secondary outcome). All pulmonary resections were performed at a single institution by one of five attending surgeons through an anterior muscle sparing thoracotomy or a videoassisted thoracoscopy. Stapler devices were utilized for division of incomplete fissures and closing the bronchus. A mediastinal systematic sampling or complete dissection was performed in patients with lung cancer. Air leak was tested at $20-30 \mathrm{cmH}_{2} \mathrm{O}$ pressure by pouring isotonic to pleural space after resection in patients who underwent pulmonary resection through video-thoracoscopic vision or anterior or posterolateral thoracotomy. During reinflation of the remaining lung, air leak was assessed. If no air leak was observed, the patient was not included. After water submersion test for air leaks, the surface of the cut parenchyma was dried. Patients with air leak were randomized to one of two groups: Homologous-fibrin sealant group in which $8 \mathrm{~mL}$ of homologous fibrin $\left(\right.$ Tiesse $^{\circledR}$ ) was implemented, or autologous group in which $7 \mathrm{~mL}$ autologous fibrin sealant (Vivostat ${ }^{\circledR}$ ) prepared from the patient's blood (approx. $300 \mathrm{~mL}$ ) in accordance with the standard application guide.

Autologous sealant Vivostat was prepared using the 'Vivostat system' which is an automated device for the perioperative preparation of a patient-derived fibrin material. An amount of $150 \mathrm{~mL}$ of the patient's blood was retrieved via a previously applied arterial access line after the resectability was confirmed in patients whom pneumonectomy was not required and whom there was air leak after the planned resection. Blood was collected into the citrate-containing cylinder. The preparation cylinder was then placed within the processor unit. System processed the blood for 20 minutes to produce autologous fibrin solution. The solution was placed in the application unit to be applied with a hand-held spray-pen device. The insufflation was performed after 2 minutes for 


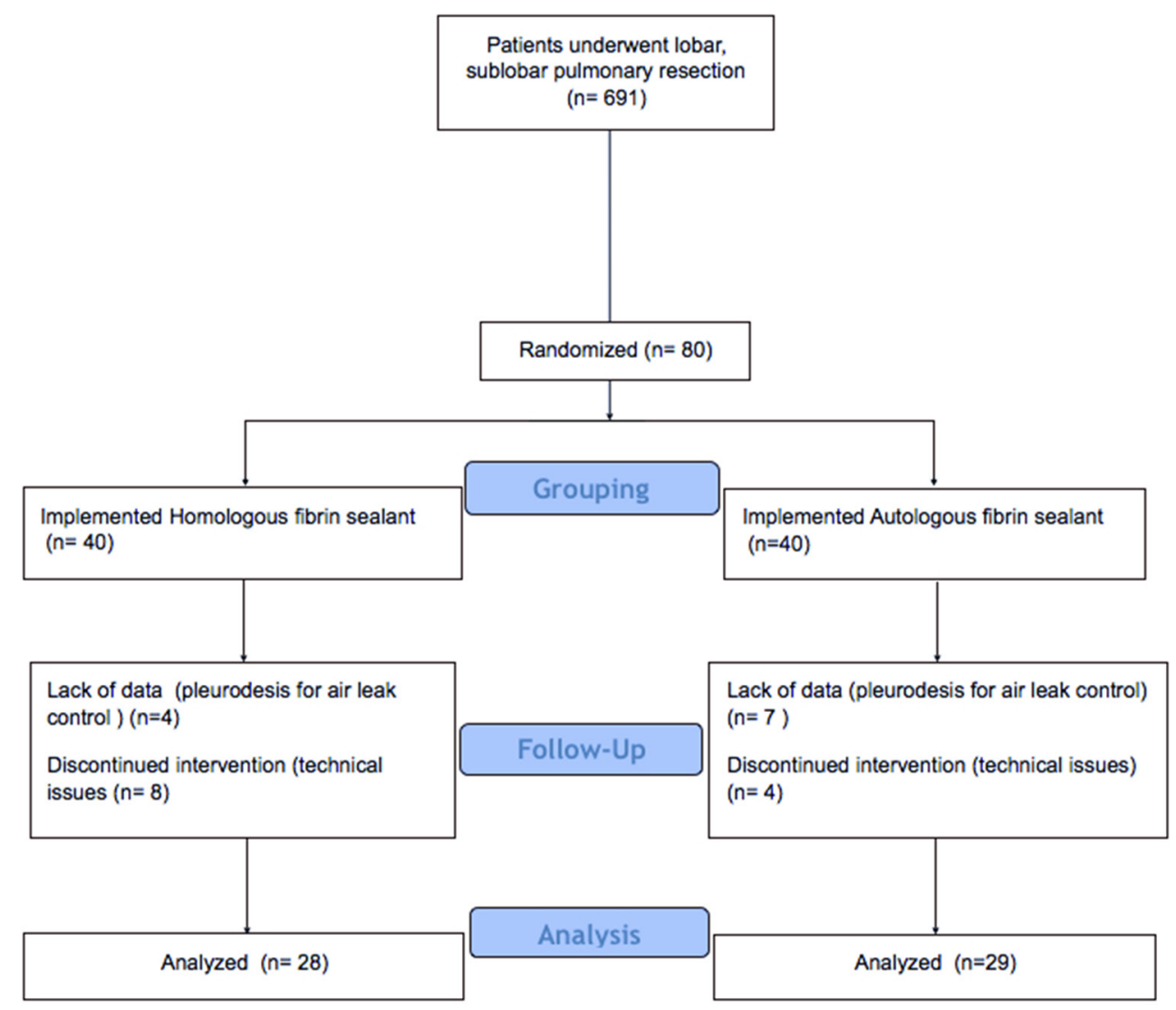

Figure 1 Consort diagram of the study.

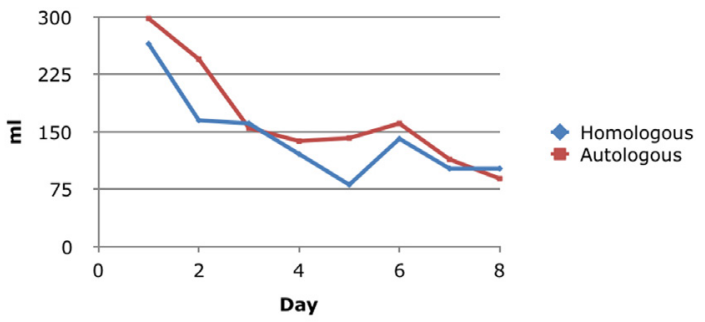

Figure 2 Daily fluid drainage of drain (mL).

allowing polymerization. Two minutes were allowed before insufflation of the lung to allow polymerization. Tisseel was prepared by reconstitution of sealer protein concentrate and thrombin in fibrinolysis inhibitor solution and calcium chloride solution. The sealer protein solution and thrombin solution are then merged using an Application system called 'Duploject'. Tisseel was then applied using a spray device.

In both groups, the sealant was administered on the suture lines by spraying. Patients were implemented early removal of chest tube protocol with the digital system $\left(\right.$ Thopaz $\left.^{\circledR}\right)$ that continuously controls the air leak. All patients sustained digital device for air leak monitoring during whole postoperative period until the drain was removed. The system has measured the air outputs from thorax tubes to drain and the pressure inside the thorax and recorded them to its memory unit available in its own system. After the drains were removed, the data in the digital measurement devices were transferred to computer and the mean values were calculated. The primary outcome measure was rate of prolonged air leak and TRT was secondary outcome parameter. We also analyze the hospital stay of the patients as an important clinical parameter. The final pathological diagnoses of patients included lung carcinoma in 42 patients $(73.6 \%)$, lung metastasis in 5 patients $(8.7 \%)$ and benign disease in 10 patients (17.7\%). Patients excluded from the study were those who underwent pneumonectomy, the ones who did not want to take part in the study and the patients who did not have any leak in the leak control during surgery. The air leak 
follow-up was carried out through the constant $-20 \mathrm{cmH}_{2} \mathrm{O}$ pressure implemented by Thopaz ${ }^{\circledR}$ device. The chest tube was removed if the volume of drained fluid was $<300 \mathrm{~mL}$ in $24 \mathrm{~h}$; provided there was no frank blood, transpleural air flow recorded by the digital system was $<20 \mathrm{~mL} / \mathrm{min}$. The indications of drain removal were the same in two groups.

Institutional review board and Ethics Committee approved the study protocol (2011/B-16). All participants gave informed consent.

The recorded parameters were stay in the intensive care unit, days of air leak, duration of chest tube, total drainage, maximum air leak, mean air leak, duration of hospital stay, complications (subcutaneous emphysema, atelectasis, etc.) and the occurrence of chest tube re-insertion and postoperative complications developed in patients.

\section{Results}

The demographic data of the patients before surgery is shown in Table 1. There was no difference between the groups in terms of age, gender, $\mathrm{FEV}_{1}, \mathrm{FVC}$ and surgical procedures $(\mathrm{P}>0.05)$. There was no 90 -day mortality. There was no significant difference between the groups in terms of the maximum amount of leak, the mean air leak and the rate of prolonged air leak ( $>7$ days) (Table 2). The mean total amount of drainage in the homologous sealant group (Tisseel) was $643.21 \pm 586.63 \mathrm{~mL}$ while it was $780.3 \pm 512.33 \mathrm{~mL}$ $(\mathrm{P}=0.355)$ in the autologous (Vivostat) group. The drain removal time was not statistically significantly different between groups (Table 2) $(\mathrm{P}=0.978)$. Similarly, mean hospital stays were similar between groups $(\mathrm{P}=0.140)$. Median duration of hospital stay was 5 days in two groups. There was no significant difference between the groups in terms of complication rates $(\mathrm{P}=0.711)$ (Table 2$)$.

There were five patients $(8.7 \%)$ who had prolonged air leak in both groups $(\mathrm{P}=0,669)$. A significant relationship was not found between prolonged air leak and clinical parameters $(\mathrm{P}>0.05)$.

When patients who underwent lobectomy were analyzed, mean air leak was $85.6 \mathrm{~mL} / \mathrm{min}$ in the homologous group, whereas it was $31.5 \mathrm{~mL} / \mathrm{min}$ in the autologous group $(\mathrm{P}=0.069)$. In patients who had undergone wedge resection, mean air leaks were 890 and $697 \mathrm{~mL} / \mathrm{min}$ in homologous and autologous sealant groups respectively $(\mathrm{P}=0.752)$. A statistically significant difference was also not determined between the groups in terms of maximum amount of leak $(\mathrm{P}=0.950)$. In patients who underwent lobectomy, the total amount of drainage was $841.6 \mathrm{~mL}$ in homologous sealant arm, and $737.5 \mathrm{~mL}$ in autologous sealant arm $(\mathrm{P}=0.783)$. In this lobectomy subgroup, the mean of maximum air leak was $795.89 \mathrm{~mL} / \mathrm{min}$ heterologous group and it was $717.44 \mathrm{~mL} / \mathrm{min}$ in the homologous group $(\mathrm{P}=0.833)$. The mean air leak were 91.22 and $52.8 \mathrm{~mL} / \mathrm{min}$ in heterologous and homologous groups respectively $(\mathrm{P}=0.324)$. When the patients who underwent upper lobectomy were analyzed, mean air leak were 62.3 and $26.9 \mathrm{~mL} / \mathrm{min}$ in homologous and autologous sealant groups respectively $(\mathrm{P}=0.224)$. Complications developed in 13 patients in the homologous group, whereas 11 patients had at least one postoperative complication in autologous group (Table 2). There was no statistically significant difference in terms of developed complications $(\mathrm{P}=0.711)$.

\section{Discussion}

In this study, there was no difference in the efficacy of homologous and autologous sealants in the control of postoperative air leak in patients who underwent pulmonary resection.

As the most frequent morbidity in thoracic surgery, air leak is dealt with different strategies. Belda-Sanchís (6) indicated that with a Cochrane library systematic review, surgical sealants has been found be effective to reduce postoperative air leaks and time to chest tube drainage time however, they are not associated with a less hospital stay. It has been also demonstrated that, a sealant patch can reduce the duration of air leak (2).

Air leak in especially patients with emphysematous lungs may continue longer than 7 days (7). All efforts should be exerted to prevent, reduce and avoid from postoperative air leak. In our study, although, homologous sealant provided less air leak, slightly shorter drainage time, the differences were not statistically significant.

In their study, Brunelli et al. found that, the rate of air leak and intrapleural pressure difference as surrogate for the air leak that will continue for $72 \mathrm{~h}$ independently of the other variables (8). In this study, we did not find intrapleural pressure difference as a predictor of prolonged air leak. Cerfolio et al. (9) especially emphasized that the best and easiest way to treat air leak was to prevent air leak during operation. It was shown that the use of fibrin glue reduced the complications such as pneumothorax and secondary infections and prolonged air leak $(10,11)$. In our study, the total rate of complications determined in both groups was acceptably low and there was no significant difference between the groups. Belcher and colleagues (12) reported 
Table 1 Characteristics of the patients

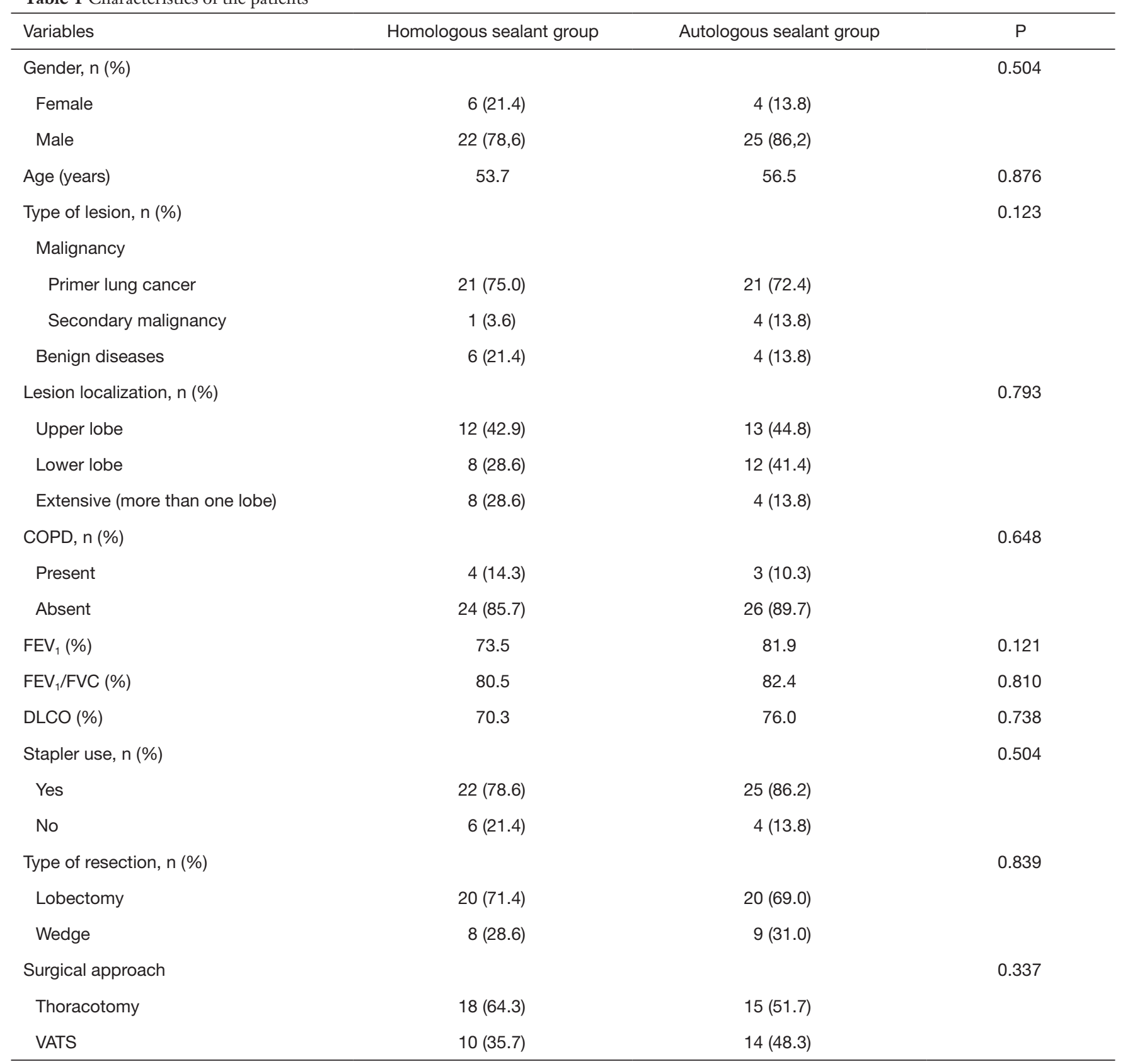

VATS, video assisted thoracoscopic surgery.

that, clinical pleural space infection were $2 \%$ and $8 \%$ in their patients who had Vivostat and Bioglue. We did not observe any pleural infection in our series.

Fabian et al. concluded that the use of fibrin glue in pulmonary resections significantly reduced prolonged air leak (13). In our study, a low rate of prolonged air leak was seen in both groups, while the amount of leak seen on the 7th day was higher in the homologous sealant group. However, the difference was not statistically different. We also did not notice any different effect of autologous sealant in terms of chest tube drainage, air leak time, hospital stay when we compare the parameters of those in homologous sealant group. We also did not find a statistically significant difference in maximal or mean air leak between groups. 
Table 2 Comparison between the study groups in terms of postoperative measurements

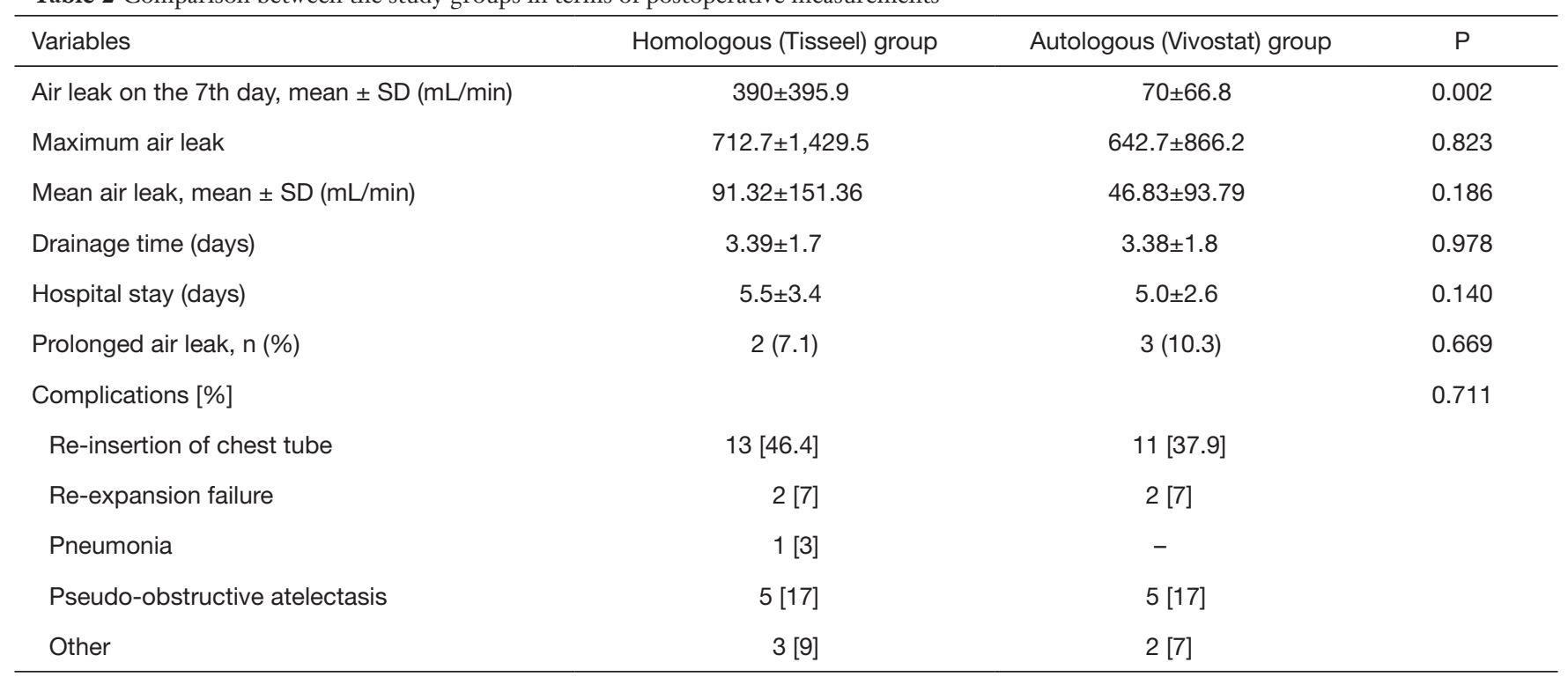

SD, standard deviation.

Buchta et al. concluded that there was a significant difference between the fibrin concentrations created by autologous and homologous fibrin glues and clotting times (14). For homologous sealants, there is a risk of antigenic reactions since used sealant proteins are slightly different (15). However, in this study, we did not see a complication related to allergic reaction or infection in any of the patients in the homologous sealant (i.e., Tisseel) group. The autologous properties of Vivostat reduce the theoretical potential for foreign tissue reaction and does not possess the possible concerns regarding viral infection. Nevertheless Tisseel has been produced excluding the any viral contamination. Vivostat was reported to have extensive elastic properties and 5 days of absorption time shown in animal models (16). The disadvantages of the Vivostat include time necessary for the preparation of study and necessity of withdrawing approximately $300 \mathrm{~mL}$ of blood. Nevertheless we did not observe any hemodynamic instability after blood removal since the amount is well below the tolerable blood loss.

In their retrospective study that involves 360 patients, among whom 102 patients were implemented fibrin glue during surgery, Gagarine et al. (17) reported a reduction of 1.4 days in the chest tube time in the fibrin glue group comparing to the control group, but they did not find a significant difference in terms of prolonged air leak and duration of hospital stay (6.3-7.7 days). Petrella and colleagues also reported that, another agent Innoseal might shorten the air leak time in patients undergoing resections for metastasis (18). In our series, five patients had metastasis. However, we have number of patients with primary tumor or benign lung disease. Using a different air leak sealant, Marta et al. observed that, TachoSil provided a statistically significant reduction in intra-operative air leak (19). However, median time until chest drain removal was 4 days with TachoSil and 5 days in the standard group. There was no difference between groups in hospital length of stay (19).

In our study, we used a digital chest drainage device which is capable of continuously recording and monitoring the amount of air leak. The device provides objective information about air leak, and helps to create reproducible chest tube protocols while minimizing the inter-observer variability in decision making process of removing chest tubes (2). Brunelli and colleagues indicated that (4), the application of a chest removal protocol using the device was safe and cost-effective. For this reason, we used the digital system in our study. We also compared the maximum and mean amount of air leak in order to find a possible surrogate of shorter chest tube drainage time. However, we did not find a statistically significant difference in chest tube removal and hospital stay between two groups. Nevertheless, usage of system may theoretically reduce the random effects which might have played a role in our study in terms of chest tube management. 
Belcher and colleagues reported that, the incidence of clinical pleural infection was $8 \%$ in the patients receiving BioGlue, $2 \%$ in the Vivostat arm (12). We observed 24 complications in 57 patients (42.1\%). However, none of them was infectious complication. For this reason, it is plausible to comment that, homologous or autologous sealants did not increase the incidence of pleural infection following resection.

Since FDA approved homologous fibrin sealant for air leak prevention, we aimed to compare the autologous agent in order to find if it is equally effective in patients undergoing pulmonary resections. The homologous system has advantages of quick preparation, price and no requirement of special device of fibrin separation from whole blood.

Limitations of the study should be addressed. We studied in a relatively small number group of patients. However, the sample size was calculated to be adequate for showing a possible difference at a power of 0.9 . We also did not proceed a long term follow-up in the study since no long term side-effects or complication can be expected.

\section{Conclusions}

In this study, the air leak and the rate of prolonged air leak occurrences could be reduced to quite lower levels with the use of fibrin products. Homologous and autologous fibrin sealants seem to be equally effective in terms of duration of hospital stay, prevention of prolonged air leak. Further studies are warranted in order to test or confirm our findings.

\section{Acknowledgements}

None.

\section{Footnote}

Conflicts of Interest: The authors have no conflicts of interest to declare.

Ethical Statement: Institutional review board and Ethics Committee approved the study protocol (2011/B-16). All participants gave informed consent.

\section{References}

1. Varela G, Jiménez MF, Novoa N, et al. Estimating hospital costs attributable to prolonged air leak in pulmonary lobectomy. Eur J Cardiothorac Surg 2005;27:329-33.

2. Lang G, Csekeö A, Stamatis G, et al. Efficacy and safety of topical application of human fibrinogen/thrombincoated collagen patch (TachoComb) for treatment of air leakage after standard lobectomy. Eur J Cardiothorac Surg 2004;25:160-6.

3. Spotnitz WD, Prabhu R, Welker R, et al. Clinical uses of fibrin sealant. In: Mintz PD. editor. Transfusion therapy: clinical principles and practice. 2nd ed. Bethesda: AABB Press, 2004:437-77.

4. Brunelli A, Salati M, Refai M, et al. Evaluation of a new chest tube removal protocol using digital air leak monitoring after lobectomy: a prospective randomised trial. Eur J Cardiothorac Surg 2010;37:56-60.

5. Dernevik L, Belboul A, Rådberg G. Initial experience with the world's first digital drainage system. The benefits of recording air leaks with graphic representation. Eur J Cardiothorac Surg 2007;31:209-13.

6. Belda-Sanchís J, Serra-Mitjans M, Iglesias Sentis M, et al. Surgical sealant for preventing air leaks after pulmonary resections in patients with lung cancer. Cochrane Database Syst Rev 2010;1:CD003051.

7. Potgieter PD, Benatar SR, Hewitson RP, et al. Surgical treatment of bullous lung disease. Thorax 1981;36:885-90.

8. Brunelli A, Cassivi SD, Salati M, et al. Digital measurements of air leak flow and intrapleural pressures in the immediate postoperative period predict risk of prolonged air leak after pulmonary lobectomy. Eur J Cardiothorac Surg 2011;39:584-8.

9. Cerfolio RJ, Tummala RP, Holman WL, et al. A prospective algorithm for the management of air leaks after pulmonary resection. Ann Thorac Surg 1998;66:1726-31.

10. Mouritzen C, Drömer M, Keinecke HO. The effect of fibrin glueing to seal bronchial and alveolar leakages after pulmonary resections and decortications. Eur J Cardiothorac Surg 1993;7:75-80.

11. Petsas T, Siamblis D, Giannakenas C, et al. Fibrin glue for sealing the needle track in fine-needle percutaneous lung biopsy using a coaxial system: Part II--Clinical study. Cardiovasc Intervent Radiol 1995;18:378-82.

12. Belcher E, Dusmet $M$, Jordan S, et al. A prospective, randomized trial comparing BioGlue and Vivostat for the control of alveolar air leak. J Thorac Cardiovasc Surg 2010;140:32-8.

13. Fabian T, Federico JA, Ponn RB. Fibrin glue in pulmonary resection: a prospective, randomized, blinded study. Ann Thorac Surg 2003;75:1587-92. 
14. Buchta C, Hedrich HC, Macher M, et al. Biochemical characterization of autologous fibrin sealants produced by CryoSeal and Vivostat in comparison to the homologous fibrin sealant product Tissucol/Tisseel. Biomaterials 2005;26:6233-41.

15. Cederholm-Williams SA. Fibrin glue. BMJ 1994;308:1570.

16. Velada JL, Hollingsbee DA. Physical characteristics of Vivostat patient-derived sealant. Implications for clinical use. Eur Surg Res 2001;33:399-404.

17. Gagarine A, Urschel JD, Miller JD, et al. Effect of fibrin

Cite this article as: Kılıç B, Erşen E, Demirkaya A, Kara HV, Alizade N, İşcan M, Kaynak K, Turna A. A prospective randomized trial comparing homologous and autologous fibrin sealants for the control of alveolar air leak. J Thorac Dis 2017;9(9):2915-2922. doi: 10.21037/jtd.2017.08.02 glue on air leak and length of hospital stay after pulmonary lobectomy. J Cardiovasc Surg (Torino) 2003;44:771-3.

18. Petrella F, Borri A, Brambilla D, et al. Efficacy and safety of Innoseal for air leak after pulmonary resection: a casecontrol study. J Surg Res. 2016;206:22-6.

19. Marta GM, Facciolo F, Ladegaard L, et al. Efficacy and safety of TachoSil@ versus standard treatment of air leakage after pulmonary lobectomy. Eur J Cardiothorac Surg 2010;38:683-9. 